\title{
Host-Pathogen Interactions Among Barley Genotypes and Bipolaris sorokiniana Isolates
}

\author{
H. Ghazvini and A. Tekauz, Cereal Research Centre, Agriculture and Agri-Food Canada, Winnipeg, MB R3T 2M9, \\ Canada
}

\begin{abstract}
Ghazvini, H., and Tekauz, A. 2008. Host-pathogen interactions among barley genotypes and Bipolaris sorokiniana isolates. Plant Dis. 92:225-233.
\end{abstract}

Spot blotch, caused by Bipolaris sorokiniana, is a damaging foliar disease of barley (Hordeum vulgare) and other cereal grains in Canada. In order to deploy resistance as an effective management tool, information on the virulence diversity present in the local population of the pathogen is necessary. Speculation regarding the presence of differential virulence versus continuous aggressiveness in $B$. sorokiniana isolates was begun when isolates with differential virulence on the barley cv. Bowman and its derivatives were reported in North Dakota. To further evaluate the $H$. vulgare-B. sorokiniana interaction model, the infection responses induced by 127 isolates of the pathogen collected from Canada and other parts of the world were analyzed on 12 differential barley lines using quantitative approaches. Analysis of the data based on infection response elicited on the barley differentials resulted in an enhanced understanding of the host-pathogen interactions and in the relationships among isolates, compared with the classical method of pathotype identification. The population of $B$. sorokiniana was determined to consist of three distinct pathogenic groups (having low virulence, differential virulence, and virulence with varying levels of aggressiveness). The results of the various quantitative approaches indicated that complex interactions exist among barley genotypes and $B$. sorokiniana isolates of the third pathogenic group which cannot be analyzed easily using the classical method of pathotype identification. It also was inferred that the gene-for-gene model is not the principal system operating in the $H$. vulgare-B. sorokiniana pathosystem, although this plays a role in some interactions.

Additional keywords: cluster analysis, regression analysis

Barley (Hordeum vulgare L.) is the second most important cereal grain in Canada and, based on the 5-year average from 2000 to 2004, occupies a land base of about 4.9 million ha (3). Annual barley production in Canada from 2000 to 2004 was around 11.4 million tons (3). Spot blotch, caused by Bipolaris sorokiniana (Sacc.) Shoemaker (teleomorph Cochliobolus sativus (S. Ito \& Kurib.) Drechsler ex Dastur, syn. Helminthosporium sativum Pammel, C. M. King \& Bakke) is one of the major foliar diseases of barley in Canada and can cause significant damage to the crop (5). The disease can be controlled

Corresponding author: A. Tekauz

E-mail: atekauz@agr.gc.ca

\section{Contribution No. 1949.}

* The $\boldsymbol{e}$-Xtra logo stands for "electronic extra" and indicates that two supplementary figures and one supplementary table not included in the print edition are available online.

Accepted for publication 23 August 2007.

doi:10.1094/PDIS-92-2-0225

(C) 2008 Department of Agriculture and Agri-Food, Government of Canada; Her Majesty the Queen in Right of Canada, as represented by the Minister of Agriculture and Agri-Food Canada by several common management strategies, including chemical seed treatment, foliar fungicide sprays, cultural practices, and host resistance.

Information on the virulence diversity of plant pathogens is necessary to deploy resistance genes against the prevalent pathotypes. Reports describing the presence of differential interactions and nondifferential interactions between host genotypes and pathogen isolates in the barley-B. sorokiniana pathosystem have been published in the literature. The first evidence of host-specific virulence in isolates of $B$. sorokiniana was reported by Levitin et al. (24). Subsequently, Fetch and Steffenson (12) reported on B. sorokiniana isolates with differential virulence on the cv. Bowman and its derivatives in North Dakota. The term "pathotype," which is the virulence phenotype of an isolate as elicited on a set of host differentials, was introduced in the $H$. vulgare-B. sorokiniana pathosystem when ValjavecGratian and Steffenson (35) evaluated the virulence diversity of 36 isolates of $B$. sorokiniana collected mostly from North Dakota and detected differential virulence using three differential lines (DLs). Valjavec-Gratian and Steffenson (34) reported that both virulence in $B$. sorokiniana isolate ND90Pr (pathotype 2) and resistance in the barley line ND 5883 were controlled by a single gene. However, they emphasized that their finding neither confirmed nor refuted a possible gene-for-gene interaction in the $H$. vulgare-B. sorokiniana pathosystem. Nonetheless, the term pathotype was used extensively to describe virulence diversity of $B$. sorokiniana populations in subsequent studies. Differential virulence in $B$. sorokiniana likewise has been reported in several other recent studies $(4,14,28)$.

Despite several recent reports of differential virulence in the $B$. sorokinianabarley pathosystem, this type of virulence has rarely been reported in isolates of $B$. sorokiniana collected from wheat cultivars. Tinline (32) stated that, because most $B$. sorokiniana isolates were virulent on wheat and displayed no differential virulence, almost any host isolate could be used in screening wheat for resistance. Likewise, Maraite et al. (27) had suggested that $B$. sorokiniana isolates, unlike the rusts, do not exhibit clear virulence patterns and consist of a continuum of strains differing in aggressiveness. Analysis of infection responses (IRs) of 12 wheat cultivars inoculated with 206 B. sorokiniana isolates collected from 24 countries showed that only 1 to $2 \%$ of the variance could be attributed to interactions between host and pathogen (17). Duveiller and Garcia Altamirano (9) suggested that the pathogen appeared to be a continuum of isolates differing in aggressiveness. Caten (8) had suggested that, in the case of no known differential interactions between host genotypes and their pathogens, isolates should be classified into "aggressive races." Bos and Parlevliet (7) described aggressiveness as whether and to what extent a pathogen can attack an organism, and Andrivon (2) defined aggressiveness as depicting quantitative, nonspecific interactions between pathogen isolates and host genotypes.

Reports of nondifferential versus differential interactions between host genotypes and $B$. sorokiniana isolates suggest that the host-pathogen interaction in this system should be studied in more detail (e.g., by using a quantitative approach to data analysis). The classical method of pathotype identification, based on IRs of 12 barley genotypes to 127 isolates of $B$. sorokiniana collected from Canada and other parts of the world, was evaluated based on the assumption that interactions between 
isolates and barley genotypes are controlled by a gene-for-gene system (15). This analysis allowed classification of the $B$. sorokiniana population into eight virulence groups (VIGs) on the basis of transformation of the 1-to-9 categorical scale of infection on DLs into binary values of resistance and susceptibility. However, intermediate IRs (near the cut-off point between resistance and susceptibility) frequently were observed among genotypes; this made it difficult to assign these types of reactions unequivocally to either the resistant or susceptible classes. Although the classical method of pathotype identification requires separation of IR values of genotypes into binary values of resistant and susceptible, a quantitative analysis of host-pathogen interactions in this pathosystem using the actual IRs is warranted. The objectives of this study were to further evaluate the $H$. vulgare $-B$. sorokiniana interaction model by using the quantitative approaches of analysis of variance (ANOVA), regression, and cluster analysis to explain the different disease IR observed on barley DLs. Averages of IR values of DLs to each isolate were considered as a continuous criterion in the analysis of the data from a quantitative point of view.

\section{MATERIALS AND METHODS}

Disease assessment and virulence group designation. Propagation of the host plants, preparation and application of inoculum, and disease assessment as well as the VIG designation based on the classical method of differentiation are described in Ghazvini and Tekauz (15). Briefly, 127 isolates of $B$. sorokiniana, including 92 Canadian isolates collected from Manitoba, Saskatchewan, Alberta, Ontario, and Quebec together with 35 exotic isolates obtained from Australia, Uruguay, Poland, Brazil, the United States, and Mexico, were used to evaluate host-pathogen interactions in the barley-B. sorokiniana pathosystem. A differential set of 12 barley lines or cultivars, including 6 Canadian genotypes (TR 251, TR 261, Newdale, CDC Stratus, CDC Bold, and AC Metcalfe) and 6 genotypes from the United States (ND B112, ND 5883, Bowman, Conlon, Robust, and Stander) was used to test pathogen isolates. Differential lines were seeded 2 weeks prior to inoculation as four clumps of eight seeds per 30-cm-diameter clay pot. Plants were grown in a controlled environment cabinet (Conviron Model PGW36; Controlled Environment Inc., Winnipeg, Manitoba, Canada) at $17^{\circ} \mathrm{C}$ and with an 18-h photoperiod. Aqueous conidial suspensions of each isolate were prepared from single spore cultures. The conidial concentrations were adjusted to 3,000 conidia/ml by adding doubledistilled water to each sample. A drop of polyoxyethylene-20-sorbitan monolaurate (Tween 20) was added per $50 \mathrm{ml}$ of sus- pension as a spreader and sticker to facilitate adherence and the even distribution of inoculum. Sufficient inoculum was applied to leaves of 2-week-old seedlings to cause run-off, approximately $10 \mathrm{ml}$ per seedling clump. Inoculated plants were incubated in darkness for $18 \mathrm{~h}$ at 20 to $22^{\circ} \mathrm{C}$ and $100 \%$ relative humidity. They then were grown as previously in growth cabinets at $17^{\circ} \mathrm{C}$. The second leaf of seedlings was scored for IR 10 to 12 days after inoculation using the 1 to-9 IR scale for spot blotch developed by Fetch and Steffenson (13). All isolates were tested at least twice, and some were tested three, four, or five times (15). In general, the lesions exhibited by the differential lines to different isolates of $B$. sorokiniana varied continuously. Often, the chlorotic halos surrounding necrotic lesions were not as large or uniform as illustrated by Fetch and Steffenson (13); therefore, the size of the necrotic portion of a lesion was used as the main criterion to classify IRs. The mean IR for each replication was calculated based on two to three ratings done within each clump for individual isolates, and the average IR for each isolate then was calculated across replications; this value was used for further analyses.

Data analysis. ANOVA. ANOVA was conducted in a one-way ANOVA method to test responses of DLs to each isolate separately, considering each test as an unbalanced completely randomized design. Bartlett's homogeneity of variance test (31) was used to examine the homogeneity of error mean squares of separate tests. Because of the significant differences among error mean squares, an arcsine transformation (31) was applied to the data to provide homogeneous error mean squares. A combined analysis of variance was carried out on the transformed data to determine whether there were differences between isolates in their virulence and between genotypes in their partial resistance, and also to examine the significance of genotype-isolate interactions. Data were analyzed as a two-way unbalanced design with PROC GLM using SAS software. Based on estimators of variance components, appropriate $F$ tests were conducted.

Hierarchical cluster analysis. To determine the relative virulence distances between isolates and evaluate the intra- and interrelationship among isolates of each VIG, cluster analysis was conducted using the average IR value of the DLs to each isolate. A similarity matrix based on the average taxonomic distance coefficient (30) was constructed using the SIMINT program in the NTSYS-pc software (version 2.1; Exeter Software, Setauket, NY). The similarity matrix generated then was examined using unweighted pair group method with arithmetic averages (UPGMA) clustering analysis (30) of the NTSYS-pc (SHAN module).
Regression analysis. Joint linear regression analysis (10) was used to evaluate host-isolate interactions using a quantitative approach. The average IRs of barley genotypes to different $B$. sorokiniana isolates were used to determine the degree of pathogenicity of isolates, as well as the resistant or susceptible responses of the barley genotypes. To adapt the regression analysis of Eberhart and Russell (10) for analysis of the host-pathogen interaction in the present study, each pathogen isolate was considered to be a separate environment to which the host plants were exposed and, in turn, each host genotype was considered to be a separate environment for the pathogen isolates, as described by Hamid et al. (16). Regression parameters were calculated based on splitting the interaction sum of squares (SS) into two components which represent the heterogeneity of linear regression coefficients $\left(b_{\mathrm{i}}\right)$ and the pooled deviations from individual regression lines (deviations MS). The slope of the regression for each isolate was considered to represent the aggressiveness of the isolates to increased general susceptibility of the DLs and the deviations from the regression as representing specificity of the isolate virulence with respect to the DLs (23). To evaluate the resistant responses of DLs to the isolates, the slope of the regression for each genotype was considered to represent the sensitivity of the genotypes to increased general virulence, whereas deviations from regression represented the specificity of a genotype's resistance with respect to the pathogen isolates (23).

The following linear regression model described by Eberhart and Russell (10) was used to analyze the data: $Y_{i j}=\mu_{i}+b_{i} \mathrm{I}_{j}$ $+\delta_{i j}$, where, in this study, $\mathrm{Y}_{i j}$ is the mean disease virulence/IR of the $i$ th isolate/DL on the $j$ th DL/isolate, $\mu_{i}$ is the mean disease virulence/IR of the $i$ th isolate/DL over all DLs/isolates, $b_{i}$ is the regression coefficient that measures the response of the $i$ th isolate/DL to varying levels of IR in DLs/virulence in isolates, $\mathrm{I}_{j}$ is the genotype disease IR index/isolate virulence index (environmental index) which is defined as the mean IR of all DLs/mean disease virulence of all isolates at the $j$ th isolate/DL minus the grand mean, and $\delta_{i j}$ is the sum of deviations from regression of the $i$ th isolate/DL on the $j$ th DL/isolate. Regression parameters were calculated using PROC REG using SAS software.

\section{RESULTS}

ANOVA. ANOVA identified significant differences among DLs, isolates, and isolate-differential interactions (Table 1). The largest differences were observed among DLs, indicating that the set of differentials chosen was useful in revealing virulence diversity. Significant differences among the isolates indicated that isolates differed in their virulence. Isolate-differential in- 
teractions also were a significant source of variation, suggesting that isolates responded differently to DLs. Results for the SS, a measure of variation, indicated that isolates and DLs with an SS of 79.43 and 52.41, respectively, contributed the largest portion of the total variation. Isolatedifferential interactions with an SS of 37.25 contributed a smaller portion of the total variation (Table 1 ).

Hierarchical cluster analysis of isolate virulence. Cluster analysis illustrating the virulence of isolates over DLs was done to identify relationships among isolates of each designated VIG (Fig. 1). With few exceptions, cluster analysis supported the results obtained by the classical method of VIG identification (15). Virulence variation within VIGs, as well as virulence variation between groups, was observed for isolates of all VIGs (Fig. 1). Based on cluster analysis, the eight VIGs could be separated into four major clusters (Fig. 1). Isolates of VIG 0.0.0.0 were distinct from all other groups and comprised a unique cluster of isolates with low virulence on all DLs. Isolates of VIG 6.0.0.0 composed the second major cluster and appeared to be related more closely to isolates of VIG 0.0.0.0 than to the other groups. In fact, the three isolates of VIG 6.0.0.0 identified in this study had low virulence on 10 of the 12 DLs, the exceptions being the cv. Bowman and its derivative, CDC Bold (15).

A greater similarity was found among six VIGs-6.3.5.0, 7.7.5.1, 7.7.7.5, 5.7.0.0, 5.7.4.0, and 7.7.5.4 - compared with VIGs 0.0.0.0 and 6.0.0.0 (Fig. 1). The 14 isolates designated in VIGs 7.7.7.5, 7.7.5.1, and 6.3.5.0 composed a cluster of isolates that was distinct from isolates of VIGs 0.0.0.0 and 6.0.0.0 and those of VIGs 5.7.0.0, 5.7.4.0, and 7.7.5.4. A fourth major cluster included all isolates of VIGs 5.7.0.0, 5.7.4.0, and 7.7.5.4, in which isolates of VIGs 7.7.5.4 and 5.7.4.0 formed a cluster with more similarity that, in turn, was joined to a cluster consisting of isolates of VIG 5.7.0.0 (Fig. 1).

Regression analysis of isolate virulence. For each isolate, the regression parameters were calculated and plotted against mean virulence scores of isolates to illustrate the isolate virulence on different genotypes (Fig. 2A and B). On average, all isolates in VIGs 7.7.5.4, 5.7.4.0, and 5.7.0.0 had higher regression coefficients that were distinct from those of other VIGs. For isolates of these three groups, the steeper regression slopes indicate higher isolate virulences on susceptible genotypes and lower virulences on resistant genotypes, as would be expected. In contrast, isolates of other virulence groups had lower regression slopes compared with isolates of the three groups above (Fig. 2A). Isolates of VIGs 7.7.7.5, 7.7.5.1, and 6.3.5.0 composed a cluster of isolates with higher mean disease IRs, similar to those of VIGs 7.7.5.4, 5.7.4.0, and 5.7.0.0., but their lower regression coefficients distinguished them from these isolates (Fig. 2A). The lesser slopes of isolates of VIGs 7.7.7.5, 7.7.5.1, and 6.3.5.0 indicated their higher virulence on resistant genotypes or lower virulence on susceptible genotypes. This type of virulence pattern was distinguishably different from the virulence induced by isolates of VIGs 7.7.5.4, 5.7.4.0, and 5.7.0.0. Similarly, isolates of VIGs 6.0.0.0 and 0.0.0.0, having lower regression coefficients and mean disease IRs, were distinct from other groups and from one another (Fig. 2A). All isolates of VIG 0.0.0.0 had small regression coefficients, indicating that the DLs responded in a similar way to the low virulence group of isolates. A few individual isolates with steeper slopes, similar to those that were found for VIGs 6.3.5.0, 7.7.5.1, 7.7.7.5, and 6.0.0.0, also were present among isolates of VIG 0.0.0.0 (Fig. 2A). However, the lower mean disease virulence of these isolates clearly distinguished them from isolates of other VIGs (Fig. 2A).

Deviations MS appeared not to be correlated to pathogenicity factors, except in VIG 6.0.0.0 (Fig. 2B). This parameter was distributed uniformly among isolates of all other VIGs, without showing any strong correlation to isolate virulence behavior. The higher virulence of isolates of VIG 6.0.0.0 on only two differential cultivars, Bowman and CDC Bold, and near universal low virulence on all other DLs, likely explains this wider degree of deviations MS. The higher virulence fluctuations of the three isolates of VIG 6.0.0.0 on the differential genotypes can be interpreted to result from specific gene-for-gene interactions with the DLs.

Regression analysis of differential line infection responses. For completeness, regression parameters also were calculated to evaluate the response of the DLs to the increasing virulence in the $B$. sorokiniana isolates. The mean IRs of DLs were regressed on isolate virulence indices to compare the varying degree of resistance existing among DLs (Fig. 3). Regression parameters of DLs were plotted against their mean disease IR to evaluate the summary of DL responses to the different levels of virulence found in isolates (Fig. $4 \mathrm{~A}$ and $\mathrm{B})$.

TR 251, Newdale, Stander, ND B112, and Bowman had lower regression coefficients compared with other DLs (Fig. 3). The lower regression slopes of these DLs may be an indication that such genotypes had relatively lower changes in their IRs when inoculated with weakly and highly virulent isolates of $B$. sorokiniana. By contrast, TR 261, Robust, AC Metcalfe, Conlon, CDC Bold, CDC Stratus, and ND 5883 had higher regression slopes, representing larger changes in their IRs when inoculated with weakly virulent versus highly virulent isolates (Fig. 3). Among these, ND 5883 had the highest mean disease IR and regression coefficient of any DL, representing the highest susceptibility to the most virulent isolates of $B$. sorokiniana (Fig. 4A).

Line ND 5883 and the cvs. Bowman and CDC Bold, which exhibited marked differential interactions with isolates of some VIGs (e.g., VIGs 6.0.0.0), had the highest deviations from regressions (Fig. 4B). The higher deviations MS from the regression line for these genotypes may be attributed to their differential responses (i.e., genefor-gene interaction) to specific virulences elicited by certain isolates. By contrast, some DLs, such as TR 251, TR 261, and Newdale, had reduced deviations MS, suggesting that their resistance may be largely nondifferential.

\section{DISCUSSION}

Results of the ANOVA in this study indicated significant effects for all sources of variation: isolates, DLs, and isolatedifferential interactions. Van der Plank (38) demonstrated that, in a two-variable system consisting of a host and pathogen, a highly significant interaction effect of isolate-differential in the ANOVA can be related to the virulence (differential effect) of pathogen isolates as well as the vertical (race-specific) resistance of the genotypes; it is most likely that the gene-for-gene hypothesis can be applied. He also proposed that a highly significant main effect of isolates can be related to distinct differences in aggressiveness among isolates, whereas a highly significant main effect of host genotype is indicative of horizontal (race-nonspecific) resistance among genotypes. Van der Plank $(36,37)$ defined virulence as qualitative, specific interactions between pathogen isolates and host genotypes, and aggressiveness as the quantity of disease induced by a pathogenic strain on a susceptible host. Andrivon (2) stated that most plant pathologists use and agree with these definitions for virulence and aggressiveness.

Based on these definitions, the isolates of $B$. sorokiniana evaluated in this study

Table 1. Analysis of variance of arcsine transformed mean disease virulence induced by 127 isolates of Bipolaris sorokiniana on 12 barley differential lines

\begin{tabular}{lccccc}
\hline Source of variation & df & SS $^{\mathbf{a}}$ & MS & $\boldsymbol{F}$ & $\boldsymbol{P}>\boldsymbol{F}$ \\
\hline Isolate & 126 & 79.43 & 0.630 & 93.680 & $<0.0001$ \\
Differential & 11 & 52.41 & 4.765 & 707.990 & $<0.0001$ \\
Isolate-differential & 1,386 & 37.25 & 0.027 & 3.990 & $<0.0001$ \\
Error & 2,208 & 14.86 & 0.007 & $\ldots$ & $\ldots$ \\
\hline
\end{tabular}

a Sum of squares. 


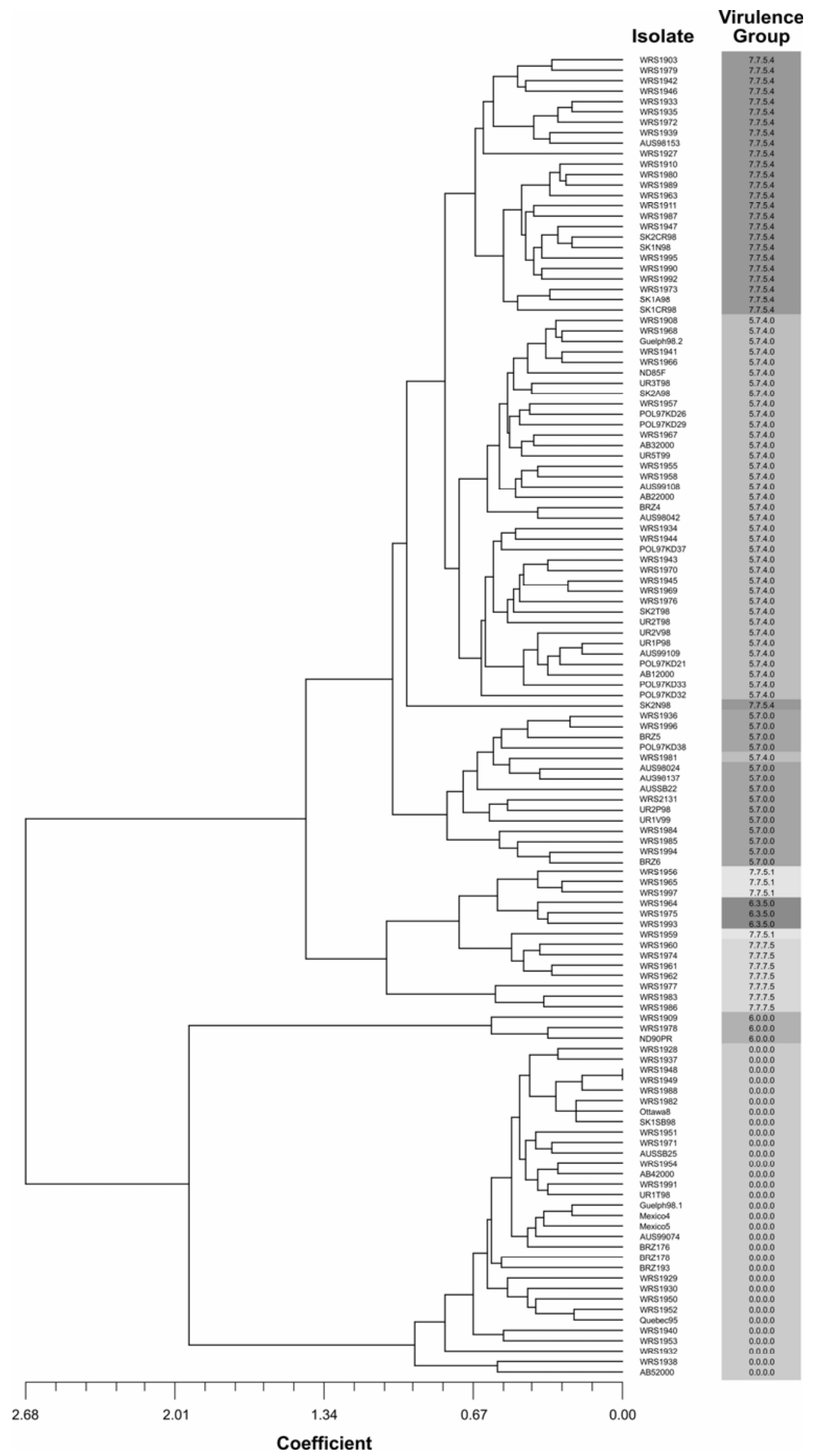

Fig. 1. Dendrogram of similarity of the mean disease virulences of 127 isolates of Bipolaris sorokiniana on 12 barley differential lines revealed by cluster analysis based on unweighted pair group method using arithmetic averages. The scale illustrated at the bottom of the dendrogram is the mean disease virulence similarity coefficient based on average taxonomic distances (30). Isolates in the same virulence group based on the classical method of virulence group designation are shading-coded. 
differed not only in their virulence but also in their aggressiveness. Van der Plank (38) proposed that pathogenicity may exist as a mixture of aggressiveness and virulence, in any proportion. Our results indicated that isolate-differential interactions were a smaller component of the total variation compared with the main effect of isolate. Therefore, it can be inferred that the aggressiveness of an isolate may be a more important factor in the $B$. sorokinianabarley pathosystem than differential virulence. However, the effect of the differential virulence of isolates, albeit smaller, indicates that a gene-for-gene relationship also is operating. Kline and Nelson (20) demonstrated that the variation among isolates of $B$. sorokiniana at a qualitative level indicated that certain genetic systems might be unique to specific isolates, whereas quantitative differences suggested a multigenic control of these systems. Further studies substantiated the views of Kline and Nelson (20), and one study (18) reported that the virulence of $B$. sorokiniana isolates to spring wheat was controlled by two genes, and to barley line ND B112 and the cv. Larker by three and four genes, respectively. By contrast, Kline and Nelson (21) found that the pathogenicity of B. sorokiniana isolates on five wild Gramineae species was conferred by a single gene. Likewise, Valjavec-Gratian and Steffenson (34) reported that a single gene controlled the virulence of isolate ND90Pr (North Dakota pathotype 2) on cv. Bowman, demonstrating a gene-for-gene interaction. ANOVA suggested that a genefor-gene system, while present, may not be the principal system operating in this hostpathogen interaction. A quantitative model for the interactions in the B. sorokinianabarley genotype pathosystem led to further cluster and regression analyses to discover the cause of significant effects for sources of variation in the ANOVA, as well as the relationships among isolates.

Results of both regression and clustering protocols indicated that isolates of $B$. sorokiniana with similar virulence patterns were aggregated into distinct clusters. A multivariate analysis of the data using principal component analysis likewise demonstrated a virulence pattern for isolates similar to those obtained by cluster and regression analyses. Tiered classification of the data indicated that the pathogen isolates could be separated into two major classes. In the first tier were those with low virulence and those with high virulence potential on at least some of the barley genotypes (e.g., VIG 6.0.0.0). Based on this major separation, isolates of VIG 0.0.0.0 were distinct from all other isolates having higher virulence potential. Both regression and cluster analysis strongly support this classification. In the second tier, the three isolates of VIG 6.0.0.0 that exhibited a gene-for-gene interaction with cv. Bowman and its derivative cv. CDC
Bold (15) could be delineated from other pathogenic isolates. The low mean disease virulence and high deviations MS of these isolates from regression slope as well as their distinct clustering in cluster analysis clearly separated them from the other pathogenic groups. The third tier comprised all isolates of the other six VIGs. However, regression and cluster analysis both showed that isolates of these six VIGs could be separated into two distinct classes. The first class includes isolates of VIGs 6.3.5.0, 7.7.5.1, and 7.7.7.5 which induced moderate virulence on most of the DLs and, thus, possessed lower regression slopes, while the second class is composed of isolates of VIGs 7.7.5.4, 5.7.4.0, and 5.7.0.0 which had higher regression slopes, representative of lower virulence on resistant and higher virulence on susceptible genotypes.

All the analytical approaches used clearly separated the $32 \mathrm{~B}$. sorokiniana isolates of VIG 0.0.0.0, having low virulence on all DLs, from all other isolates. The low virulence of Australian isolates AUSSB25 and AUS99074 on 17 barley, 1 wheat, 1 rye, and 1 triticale genotypes was reported previously by Meldrum et al. (28). These two Australian isolates also
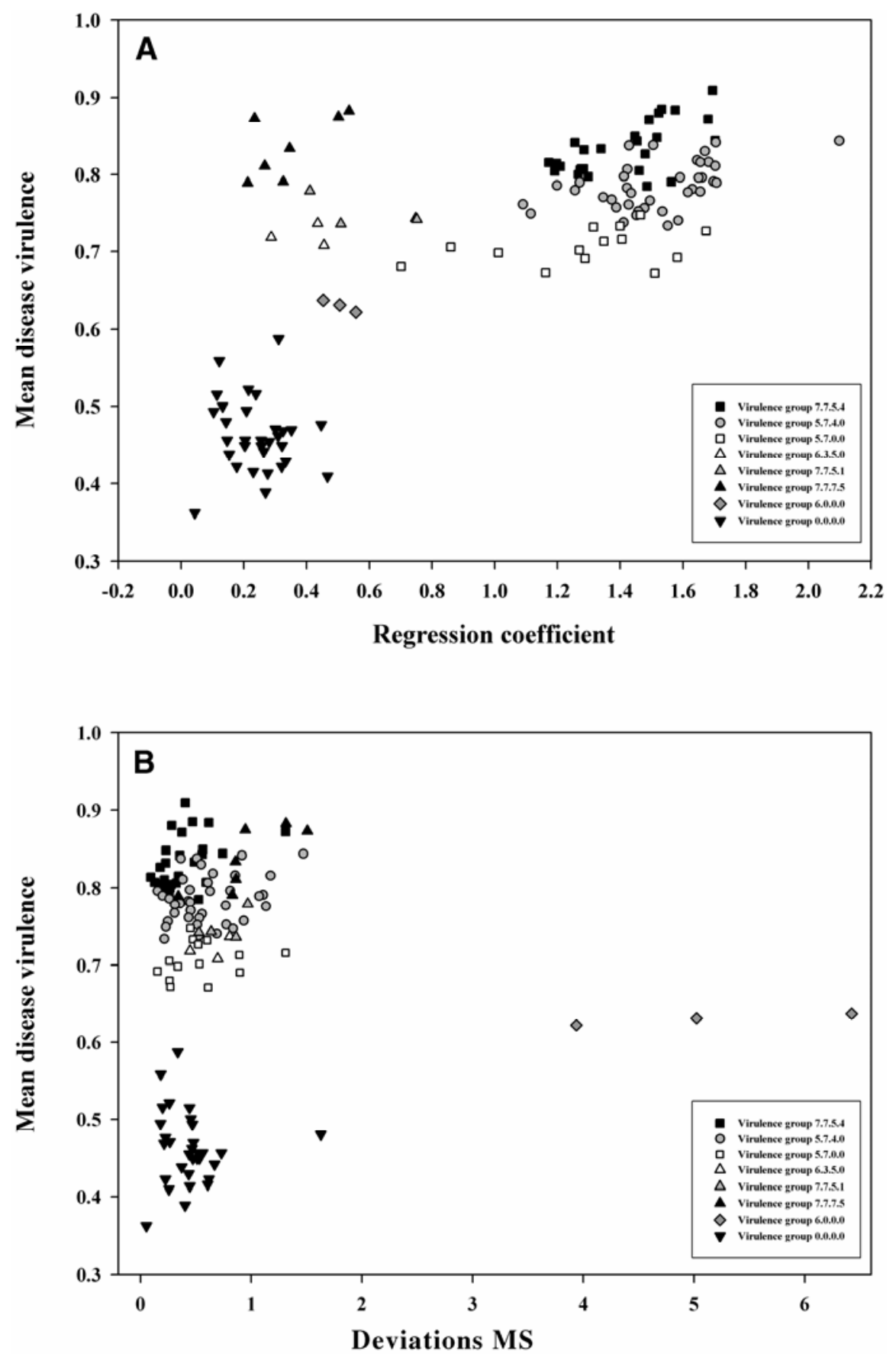

Fig. 2. Relation of mean disease virulence and the regression parameters of $\mathbf{A}$, regression coefficient and B, deviations mean squares (MS) of 127 isolates of Bipolaris sorokiniana in eight designated virulence groups. 
had low virulence on the 12 barley genotypes used in this study. They appear to be typical of low-virulence isolates of $B$. sorokiniana that were identified using a comprehensive set of very resistant to very susceptible host genotypes from Australia (28), the United States, and Canada. It is likely that resistance to these low-virulence isolates is common and universal in barley and perhaps also in other cereals. The minute foliar lesions induced on host plants by these isolates suggest that a preinfection recognition defense response is operating in host plants. This defense response against isolates of VIG 0.0.0.0 probably occurs in the cell walls and restricts penetration by the appressorium, thereby not allowing the pathogen to enter the cell, become established, and subsequently invade neighboring cells. Ibeagha et al. (19) defined the preinfection defense response as nonpenetrated cell wall appositions beneath sites of fungal attack and epidermal hypersensitive reactions, resulting in the failure of further fungal development and invasion of the mesophyll tissue.
The resistance of all barley genotypes to isolates of VIG 0.0.0.0 suggests a lack of virulence factors on barley genotypes in these isolates. This may be similar to the low-virulence race of $C$. carbonum $(H$. carbonum $=$ B. zeicola ) in corn, designated as race 0 , reported by Welz and Leonard (39). Genetic analysis indicated that isolates of race 0 in $C$. carbonum lack the virulence factors necessary to infect corn cultivars (41). It is probably preferable to avoid the use of the terms "gene-for-gene" and "specific elicitors" for $B$. sorokiniana VIG 0.0.0.0, because this assumes the presence either of many resistance genes, each found in different genotypes or species and interacting specifically with corresponding avirulent genes each found in different isolates of this group, or the presence of a universally durable resistant gene in all genotypes or species corresponding to a universal avirulent gene in all isolates of this virulence group worldwide. Both assumptions are improbable. Welz and Leonard (40) proposed that isolates of nonpathogenic race 0 in $C$. carbonum may

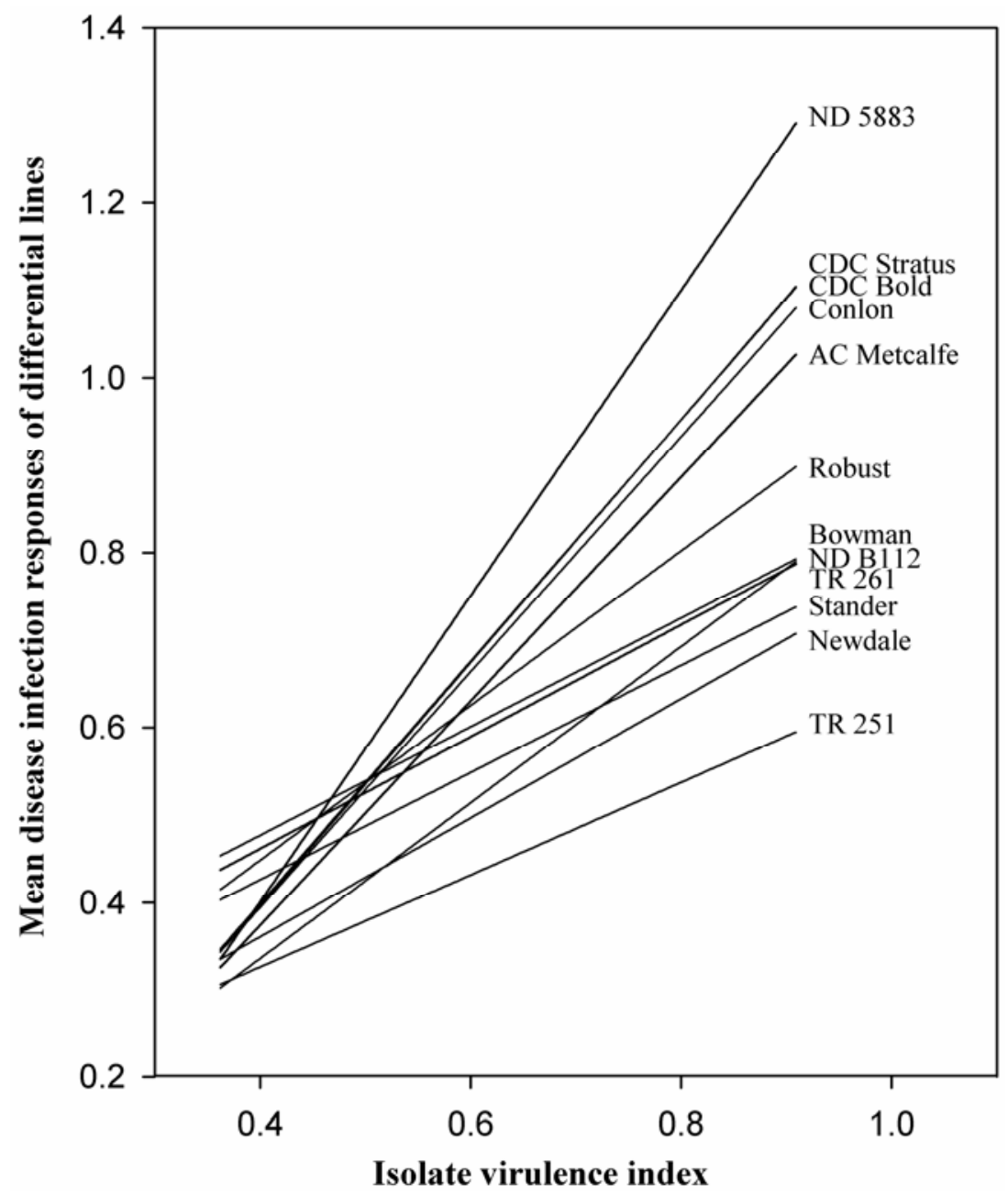

Fig. 3. Response of 12 barley differential lines to 127 isolates of Bipolaris sorokiniana with different levels of virulence or aggressiveness. The isolate virulence index for each isolate was calculated by subtracting the overall mean from the marginal mean infection responses of differentials to that isolate. The mean of the disease infection responses of each differential line were regressed on isolate virulence indexes. Regression lines of cvs. CDC Bold and CDC Stratus are overlapped and cannot be distinguished individually. have spread to corn from an unknown weed host present in farm fields. A similar scenario can be proposed for $B$. sorokiniana isolates of VIG 0.0.0.0.

In contrast to VIG 0.0.0.0, isolates of all other VIGs were found to be virulent and to express pathogenicity factors effective on at least some of the differential barley lines used. Resistance against isolates of VIG 6.0.0.0 is governed by a recessive gene (34) that probably encodes for a specific receptor to which a specific elicitor produced by this isolate type will bind. The susceptibility of the Canadian cv. CDC Bold clearly confirms the presence of a specific gene-for-gene interaction between barley lines and cultivars derived from cv. Bowman and isolates of VIG 6.0.0.0 (15). Therefore, it is appropriate to utilize the term "virulence," defined as the qualitative, specific interactions between pathogen isolates and host genotypes $(36,37)$ to separate VIG 6.0.0.0 from the other VIGs identified in this study. Kline and Nelson $(20,21)$ found that the 28 isolates of $B$. sorokiniana which had similar interactions with 26 gramineous species could be differentiated based on their pathogenicity on one to six wild species, and that pathogenicity on a given species was simply inherited and depended on one gene in five species and two genes in the sixth species. However, this type of genefor-gene interaction among isolates of VIG 6.0.0.0 and certain genotypes within a species (barley) seems to be an exception.

Excluding cv. Bowman and its derivative CDC Bold, isolates of VIG 6.0.0.0 exhibited only low virulence, similar to that of VIG 0.0.0.0, on the remainder of the DLs. The similar virulence patterns of isolates of these two VIGs suggests that isolates of VIG 6.0.0.0 might have originated from the weakly virulent isolates of VIG 0.0.0.0. Zhong et al. (43), using the chromosomes of isolate ND93.1 (North Dakota pathotype 0) as a reference, found that at least five chromosomes in isolates ND90Pr were involved in translocations. Furthermore, it also can be speculated that species-specific $B$. sorokiniana isolates of VIG 6.0.0.0 migrated from weed hosts to barley plants.

Isolates of the other six pathogenic groups induced higher IRs on a greater number of differentials than the isolates of VIG 6.0.0.0. It appears that isolates of these groups are able to overcome the host's primary defense barriers in moderately susceptible and susceptible barley genotypes. The detection of a continuous range of IRs of 2 to 9 on different barley genotypes induced by isolates of these six groups, together with frequently observed intermediate IRs of 4 and 5 among genotypes, suggest that such isolates may not demonstrate a distinct gene-for-gene interaction with differential barley lines (15). The host-pathogen interactions in isolates of these VIGs appear to follow a quantita- 
tive model in which both the pathogenicity of B. sorokiniana isolates and resistance present in barley genotypes likely are involved. Our results suggest that, for these pathogenic groups, the horizontal resistance conferred by several resistance genes in resistant host genotypes (e.g., TR 251) may provide the major inhibitory factor to thwart "successful penetration" and "successful infection," as defined by Ibeagha et al. (19). In addition, the number and expressivity of the virulence genes present in isolates of these six pathogenic groups also may affect the severity of disease and level of infection induced on the barley differentials. The reciprocal interactions of virulence genes present in pathogen isolates and resistance genes present in host genotypes may lead to the continuous range of pathogenicity observed among isolates of these six VIGs. However, this supposition is based on our limited virulence data, and additional genetic studies with both barley genotypes and high-virulence $B$. sorokiniana isolates are needed to confirm the quantitative nature of such interactions. A continuous range of pathogenicity also has been reported for $B$. sorokiniana isolates collected from wheat, and Duveiller and Garcia Altamirano (9) and Maraite et al. (27) suggested that the B. sorokiniana population on wheat appeared to be a continuum of isolates differing in aggressiveness.

Our results presented here indicate that the 14 isolates designated as VIGs 6.3.5.0, 7.7.5.1, and 7.7.7.5 were distinct from those of VIGs 7.7.5.4, 5.7.4.0, and 5.7.0.0. These 14 isolates, all from Manitoba, were moderately virulent on most of the DLs but exhibited higher virulence on the sixrowed line ND B112 and its six-rowed derivatives Robust and Stander $(29,42)$, as well as its two-rowed derivative Bowman (12). They may represent a recently evolved B. sorokiniana virulence of local origin. However, diverse levels of aggressiveness also were found among them. All isolates in this unique cluster induced reactions on the DLs which were very close to the cut-off point (IR of 4.5) between the binary designation of resistance and susceptibility (15). As such, based on their different virulences on some DLs (i.e., falling either just above or below the cutoff point), these 14 isolates likely were inappropriately separated into three VIGs by use of the classical method of classification (15). By contrast, the 78 isolates designated as VIGs 7.7.5.4, 5.7.4.0, and 5.7.0.0 had certain similarities and were classified in another major class. These isolates were found among those collected or obtained from every Canadian province sampled, as well as various other countries. On the same basis as described above (i.e., small differences in IRs on some DLs), isolates of VIGs 7.7.5.4, 5.7.4.0, and 5.7.0.0 having a high similarity were separated into three artificial VIGs using the classical method of classification (15).
Although the classical method of VIG identification, based on conversion of phenotypic IR data into the binary values of resistant and susceptible, differentiated 127 $B$. sorokiniana isolates into eight VIGs (15), the quantitative analyses of the data presented here, based on actual disease IR, provided a better means to evaluate the host-pathogen interactions in the B. sorokiniana-barley pathosystem. Even if one considers both the chlorotic and necrotic lesion components as valid measures of the IR, the 1-to-9 rating scale of Fetch and Steffenson (13) may not represent a qualitative scale (i.e., yes or no). This scale only becomes a qualitative one if categories within it are lumped together (e.g., the lumping of IRs 1 to 4 as resistant and 5 to 9 as susceptible reactions). This is what commonly has been done in several previous studies $(14,28,34,35)$ and also was used in our previous article (15), in which we applied the classical method (i.e., resistant and susceptible categories only to classify $B$. sorokiniana pathotypes). This was not the approach used here, in which each of IRs 1 to 9 was considered to be a valid entity. Used as such, the 1-to-9 scale of Fetch and Steffenson (13) is best classified as a "categorical scale" or, to be more precise, an ordinal categorical scale (1). Although a scale such as this may be con-
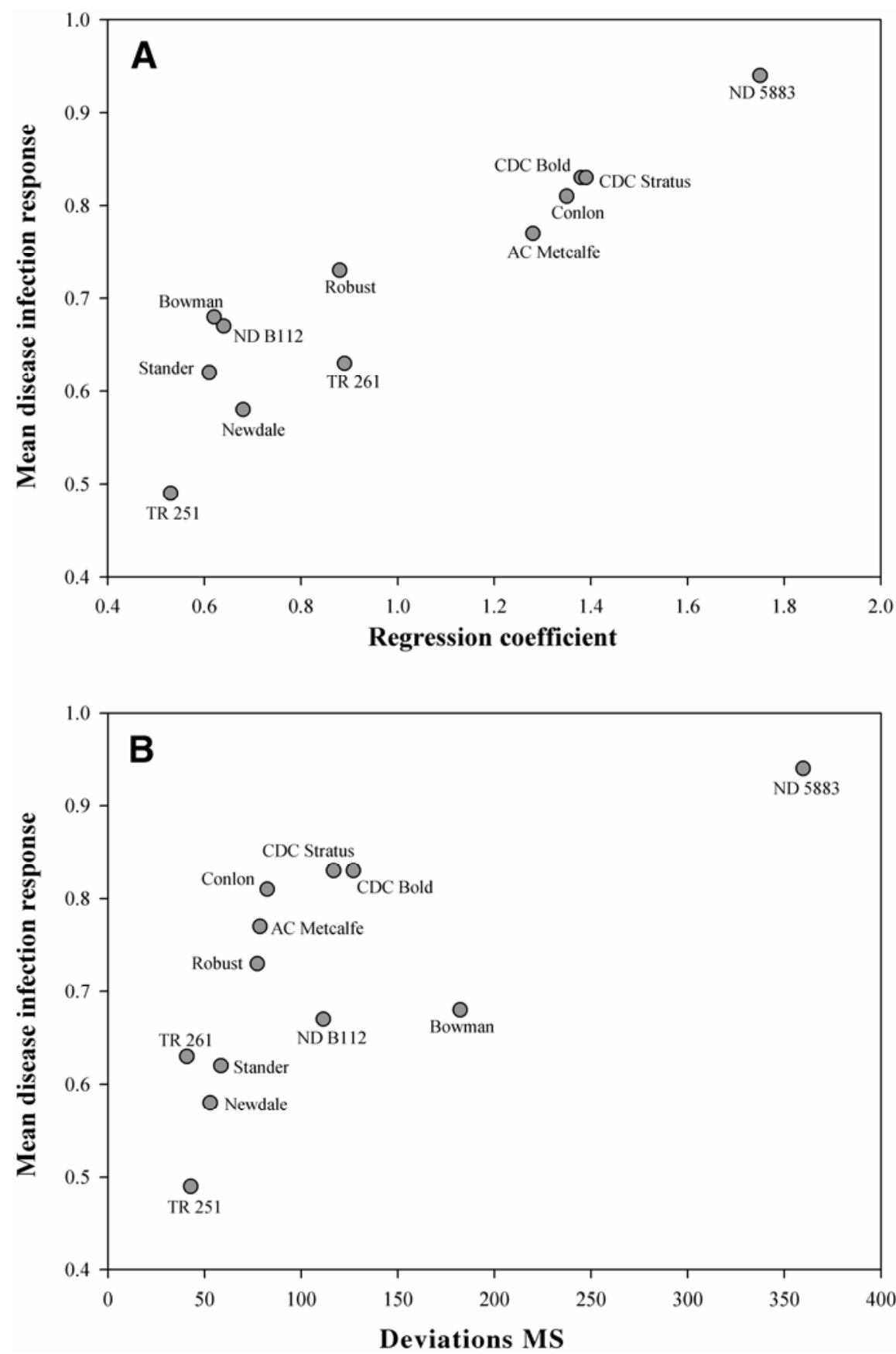

Fig. 4. Relation of mean disease infection response and the regression parameters of $\mathbf{A}$, regression coefficient and B, deviations mean squares (MS) for 12 barley differential lines inoculated with 127 isolates of Bipolaris sorokiniana. 
sidered as qualitative, because it is not based on direct measurement, it often is advantageous to treat ordinal categorical data in a quantitative manner by assigning ordered scores to the categories (1).

Such quantitative approaches also have been used by other researchers to evaluate B. sorokiniana populations. Meldrum et al. (28) used cluster analysis and regrouped the six VIGs of B. sorokiniana they identified in Australia into four distinct groups. Similarly, Arabi and Jawhar (4) used cluster analysis to classify $B$. sorokiniana isolates collected in Syria into three distinct groups based on the continuous range of IRs induced on 10 barley DLs. Likewise, regression analysis has been widely used to study host-pathogen interactions in other host-pathogen pathosystems $(11,16$, $22,23,33)$. Although the term "stability" was used in most of these studies to define either the virulence in the pathogen or resistance in the host, Van der Plank (38) was opposed to this term and argued that stability and durability are "agricultural ideals" and are best avoided when studying host-pathogen interactions. Furthermore, the joint linear regression technique itself has been regarded as an inappropriate measure of stability $(6,25,26)$. Becker and Léon (6) noted that, when applying the most appropriate biometrical method, the regression approach is of little use if the regression coefficient is included in the definition of stability. Therefore, regression analysis generally is viewed by such authors not to be a measure of stability but, rather, to provide additional information on the average response of a genotype to different environmental conditions. In our analyses, we adopted the views of Van der Plank (38) and Becker and Léon (6) and used regression parameters to provide a more comprehensive picture of the responses of both the $B$. sorokiniana isolates and the DLs interacting with one another, rather than to provide stability parameters. The results of this study suggest that joint linear regression analysis is a powerful statistical tool for the evaluation of pathosystems in which interactions are affected by both virulence and aggressiveness in the pathogen and horizontal and vertical resistance in the host.

In our previous study (15), we demonstrated that two of three North Dakota reference isolates used, ND85F and ND90Pr (pathotypes 1 and 2, respectively), exhibited a virulence pattern corresponding to isolates of virulence groups 5.7.4.0 and 6.0.0.0. However, the virulence pattern of isolate ND93-1 (representative of North Dakota low-virulence pathotype 0) was completely different from that of the lowvirulence isolates of VIG 0.0.0.0. This isolate was virulent on some differential lines in our study, including line ND 5883 previously reported to be resistant to isolate ND93-1 (35). To clarify this ambiguity, an original culture of isolate ND93-1 was obtained from Dr. Brian Steffenson, Department of Plant Pathology, University of Minnesota, St. Paul, and was retested in triplicate on the differential set together with the previously obtained isolate of ND pathotype 0 . The newly received ND93-1 isolate had low virulence on all differentials (e.g., equivalent to VIG 0.0.0.0 in our study), whereas the previously used isolate (mistakenly designated as ND93-1) still induced high virulence on some differentials. As was surmised in our previous article (15), this ambiguity could have arisen from an error in the labeling of the isolate cultures at either the original or destination laboratories. Because of practicality, we did not incorporate these new data into the already finalized analyses for this study; however, based on the low virulence pattern of "definitive" isolate ND931 on all barley differentials, in all likelihood it would have been classified with other low-virulence isolates designated as VIG 0.0.0.0.

In summary, we were able to classify 127 isolates of $B$. sorokiniana into three major groups: a low-virulence group (VIG 0.0.0.0), a pathogenic group having a specific gene-for-gene interaction (VIG 6.0.0.0), and a pathogenic group having different levels of aggressiveness (VIGs 6.3.5.0, 7.7.5.1, 7.7.7.5, 7.7.5.4, 5.7.4.0, and 5.7.0.0). This is in concordance with the groupings proposed by ValjavecGratian and Steffenson (35), who classified North Dakotan B. sorokiniana isolates into three pathotypes, designated as 0,1 , and 2. However, in our study, we found varying degrees of aggressiveness among isolates of each group. Although our VIGs 0.0.0.0 and 6.0.0.0 had virulence patterns identical to North Dakota pathotypes 0 and 2 , respectively, extensive variability was found among the other VIGs equivalent to North Dakota pathotype 1. Based on the IRs of the three DLs used, this level of variability was not reported by ValjavecGratian and Steffenson (35). Using additional DLs and isolates, Meldrum et al. (28) reported a greater diversity among pathogenic isolates of $B$. sorokiniana in Australia. We agree with the three-class grouping for the B. sorokiniana population proposed by Valjavec-Gratian and Steffenson (35), but believe that, in the case of the pathogenic groups other than VIGs 0.0.0.0 and 6.0.0.0, the considerable virulence diversity among isolates does not lend itself to incorporating all of them into a single unique pathotype group (i.e., ND pathotype 1). Our results indicated that different levels of virulence (i.e., aggressiveness) were present even among isolates of VIG 0.0.0.0 having low virulence on all DLs. Because only 3 isolates of VIG 6.0.0.0 were identified among the total of 127 tested, it was not possible to determine whether isolates of this group differed in their aggressiveness on a susceptible cultivar such as Bowman. Moreover, our find- ings demonstrate that, although the population of $B$. sorokiniana can be classified into different pathotypes, it is important to consider the virulence variation among isolates within pathotypes. Therefore, we propose that "aggressiveness" may be the most appropriate term to describe the continuous range of virulence present among isolates of each pathotype. Andrivon (2) classified pathogens into three qualitative categories-nonpathogens, avirulent pathogens, and virulent pathogens-and proposed that aggressiveness should be applied only to the latter category, as evidenced by the amount of disease produced in a particular compatible hostparasite interaction.

In conclusion, our study suggests that the gene-for-gene system likely is not the principal system operating in the $H$. vulgare- $B$. sorokiniana pathosystem, although it has the major role in specific interactions, such as those between cv. Bowman and its derivatives and isolates of VIG 6.0.0.0. The complex interactions in the $H$. vulgare $-B$. sorokiniana pathosystem cannot be analyzed easily using the simple classical method of pathotype designation, as commonly used for most biotrophic fungi with established gene-for-gene systems. The use of more sophisticated statistical techniques, such as those employed here, is preferable to adequately characterize the $B$. sorokiniana population affecting barley.

\section{ACKNOWLEDGMENTS}

We thank T. Fetch and B. McCallum, CRC$\mathrm{AAFC}$, for their proofreading and valuable comments and suggestions regarding this study; S. Woods for help provided in data analysis; and E. Mueller, M. Stulzer, and M. Beyene for their valuable technical assistance.

\section{LITERATURE CITED}

1. Agresti, A. 1996. An Introduction to Categorical Data Analysis. John Wiley \& Sons, Inc. New York.

2. Andrivon, D. 1993. Nomenclature for pathogenicity and virulence: the need for precision Phytopathology 83:889-890.

3. Anonymous. 2005. September estimates of production of principal field crops, Canada, 2005. Stat. Can. Field Crop Rep. Ser. 84:1324.

4. Arabi, M. I. E., and Jawhar, M. 2004. Identification of Cochliobolus sativus (spot blotch) isolates expressing differential virulence on barley genotypes in Syria. J. Phytopathol. 152:461-464.

5. Bailey, K. L., Gossen, B. D., Gugel, R. K., and Morrall, R. A. A. 2003. Diseases of Field Crops in Canada, 3rd ed. The Canadian Phytopathology Society, Ontario, Canada.

6. Becker, H. C., and Léon, J. 1988. Stability analysis in plant breeding. Plant Breed. 101:123.

7. Bos, L., and Parlevliet, J. E. 1995. Concepts and terminology on plant/pest relationships: Toward consensus in plant pathology and crop protection. Annu. Rev. Phytopathol. 33:69102.

8. Caten, C. E. 1987. The concept of race in plant pathology. Pages 21-37 in: Populations of Plant Pathogens: Their Dynamics and Genetics. M. S. Wolfe and C. E. Caten, eds. Blackwell Scientific Publications, Oxford. 
9. Duveiller, E., and Garcia Altamirano, I. 2000. Pathogenicity of Bipolaris sorokiniana isolates from wheat roots, leaves and grains in Mexico. Plant Pathol. 49:235-242.

10. Eberhart, S. A., and Russell, W. A. 1966. Stability parameters for comparing varieties. Crop Sci. 6:36-40.

11. Faris, M. A. 1985. Variability and interaction between alfalfa cultivars and isolates of Phytophthora megasperma. Phytopathology 75:390-394.

12. Fetch, T. G., and Steffenson, B. J. 1994. Identification of Cochliobolus sativus isolates expressing differential virulence on two-row barley genotypes from North Dakota. Can. J. Plant Pathol. 16:202-206.

13. Fetch, T. G., and Steffenson, B. J. 1999. Rating scales for assessing infection responses of barley infected with Cochliobolus sativus. Plant Dis. 83:213-217.

14. Gamba, F., and Estramill, E. 2002. Variation in virulence within Uruguayan population of Cochliobolus sativus. Pages 59-62 in: Proc. Second Int. Workshop Barley Leaf Blights. ICARDA, Aleppo, Syria.

15. Ghazvini, H., and Tekauz, A. 2007. Virulence diversity in population of Bipolaris sorokiniana. Plant Dis. 91:814-821.

16. Hamid, A. H., Ayers, J. E., and Hill, R. R., Jr. 1982. Host $\times$ isolate interactions in corn inbreds inoculated with Cochliobolus carbonum race 3. Phytopathology 72:1169-1173.

17. Hetzler, J., Eyal, Z., Mehta, Y. R., Campos, L. A., Fehrmann, H., Kushnir, U., Zekaria-Oren, J., and Cohen, L. 1991. Interactions between spot blotch (Cochliobolus sativus) and wheat cultivars. Pages 146-164 in: Wheat for the Nontraditional Warm Areas. D. A. Saunders and G. Hettel, eds. CIMMYT, Mexico D.F., Mexico.

18. Hosford, R. M., Jr., Solangi, G. R. M., and Kiesling, R. L. 1975. Inheritance in Cochliobolus sativus. Phytopathology 65:699-703.

19. Ibeagha, A., Hückelhoven, R., and Kogel, K.H. 2005. Model wheat genotypes as tools to uncover effective defense mechanisms against the hemibiotrophic fungus Bipolaris sorokiniana. Phytopathology 95:528-532.
20. Kline, D. M., and Nelson, R. R. 1963. Pathogenicity of isolates of Cochliobolus sativus from cultivated and wild gramineous hosts from the Western Hemisphere to species of the Gramineae. Plant Dis. Rep. 47:890-894.

21. Kline, D. M., and Nelson, R. R. 1971. The inheritance factors in Cochliobolus sativus conditioning lesion induction on Gramineous hosts. Phytopathology 61:1052-1054.

22. Kyong Pang, E. C., and Halloran, G. M. 1995. Adaptability and virulence specificity in Australian strains of blackleg [Leptosphaeria maculans (Desm.) Ces. et De Not.] on different host genotypes of rapeseed (Brassica napus L.). Aust. J. Agric. Res. 46:971-984

23. Leonard, K. J., and Moll, R. H. 1981. Durability of general resistance: evaluation of cultivar $\mathrm{x}$ isolate interactions. Pages 190-193 in: Proc. Symp. IX Int. Congr. Plant Prot. (Vol. I). Washington, DC.

24. Levitin, M. M., Petrova, A. N., and Afanasenko, O. S. 1985. Comparative analysis of Bipolaris sorokiniana population for virulence. Mikol. Fitopatol. 19:154-158. (In Russian)

25. Lin, C. S., and Binns, M. R. 1991. Genetic properties of four types of stability parameter. Theor. Appl. Genet. 82:505-509.

26. Lin, C. S., Binns, M. R., and Lefkovitch, L. P. 1986. Stability analysis: where do we stand? Crop Sci. 26:894-900

27. Maraite, H., Di Zinno, T., Longrée, H., Daumerie, V., and Duveiller, E. 1998. Fungi associated with foliar blight of wheat in warmer areas. Pages 293-300 in: Proc. Int. Workshop Helminthosporium Dis. Wheat: Spot Blotch and Tan Spot. E. Duveiller, H. J. Dubin, J. Reeves, and A. McNab, eds. CIMMYT, El Batán, Mexico

28. Meldrum, S. I., Ogle, H. J., and Platz, G. J. 2004. Pathotypes of Cochliobolus sativus on barley in Australia. Aust. Plant Pathol. 33:109114.

29. Rasmusson, D. C., Wilcoxson, R. D., and Wiersma, J. V. 1993. Registration of 'Stander' barley. Crop Sci. 33:1403.

30. Sneath, P. H. A., and Sokal, R. R. 1973. Numerical Taxonomy. Freeman, San Francisco.

31. Steel, R. G. D., Torrie, J. H., and Dickey, D. A
1997. Principles and Procedures of Statistics: A Biometrical Approach, 3rd ed. McGraw-Hill Inc., Boston.

32. Tinline, R D 1988. Cochliobolus sativus, pathogen of wide host range. Pages 113-122 in: Advances in Plant Pathology, Vol. 6. D. S. Ingram and P. H. Williams, eds. Academic Press, London.

33. Utkhede, R. S., and Rahe, J. E. 1983. Interactions of antagonist and pathogen in biological control of onion white rot. Phytopathology 73:890-893.

34. Valjavec-Gratian, M., and Steffenson, B. J 1997. Genetics of virulence in Cochliobolus sativus and resistance in barley. Phytopathology 87:1140-1143.

35. Valjavec-Gratian, M., and Steffenson, B. J. 1997. Pathotypes of Cochliobolus sativus on barley. Plant Dis. 81:1275-1278.

36. Van der Plank, J. E. 1963. Plant diseases: Epidemics and Control. Academic Press, New York

37. Van der Plank, J. E. 1968. Disease Resistance of Plants. Academic Press, New York.

38. Van der Plank, J. E. 1982. Host-Pathogen Interactions in Plant Diseases. Academic Press, New York.

39. Welz, G., and Leonard, K. J. 1988. Genetic variation in field populations of races 0,2 and 3 of Bipolaris zeicola in 1987. (Abstr.) Phytopathology 78:1574.

40. Welz, H. G., and Leonard, K. J. 1993. Phenotypic variation and parasitic fitness of races of Cochliobolus carbonum on corn in North Carolina. Phytopathology 83:593-601.

41. Welz, H. G., and Leonard, K. J. 1994. Genetic analysis of two race $0 \times$ race 2 crosses in Cochliobolus carbonum. Phytopathology 84:83-91.

42. Wilcoxson, R. D., Rasmusson, D. C., and Miles, M. R. 1990. Development of barley resistant to spot blotch and genetics of resistance. Plant Dis. 74:207-210.

43. Zhong, S., Steffenson, B. J., Martinez, J. P., and Ciuffetti, L M. 2002. A molecular genetic map and electrophoretic karyotype of the plant pathogenic fungus Cochliobolus sativus. Mol Plant-Microbe Interact. 15:481-492. 